\title{
Current issues and perspectives on breast cancer diagnosis
}

\author{
Shin-ichi Tsuchiya
}

Published online: 13 June 2009

(C) The Japanese Breast Cancer Society 2009

Increased incidence of breast cancer and new developments in breast cancer therapy in Japan have led to changes in procedures for the pathological diagnosis of the breast. There have been changes in the biopsy process from the conventional incisional or excisional biopsy to needle core biopsy, which acquires only minute samples, in which most pathologists currently have little experience. The diagnostic process has also seen advances because of the adoption of new methodologies, such as the immunohistochemistry of hormone receptors and HER2, not to mention the concept of triple-negative breast cancer in addition to the conventional benign-malignant diagnosis. It can therefore be said that there is a host of opportunities for tackling these new technologies.

This special edition provides commentaries from the pathologist's viewpoint on the following subjects:
(1) therapeutic strategies for breast cancer based on histological type; (2) the usefulness of immunohistochemistry on the differential diagnosis between benign and malignant breast tumor; (3) morphological characteristics of the basal-like subtype of breast carcinoma with special reference to cytopathological features; (4) gene and chromosomal alterations in sporadic breast cancer: correlation with histopathological features and implication for genesis and progression; (5) a new reporting form for breast cytology. All these reports, which feature the latest cutting-edge knowledge and concepts, come from sites where research activities are focused chiefly on the pathological diagnosis of breast cancer. Having been in charge of planning and editing, I will be more than happy if this special edition makes a contribution to clinical and pathological research centered on the breast. 